\title{
Diagnostic criteria and prevalence of mild cognitive impairment in older adults living in the community: a systematic review and meta-analysis
}

\author{
Rebeca Mendes P. Pessoa ${ }^{1}$ \\ https://orcid.org/0000-0003-0867-1821
}

ANA J ULIA L. BOMFIM2
https://orcid.org/0000-0001-7512-6343

Bianca L. Cavalmoretti Ferreira 1,3

https://orcid.org/0000-0003-3639-9626

Marcos Hortes N. Chagas $1,2,3$

https://orcid.org/0000-0003-3752-7984

'Department of Neurosciences and Behavioral Sciences, University of São Paulo (USP), São Paulo, SP, Brazil.

2 Department of Psychology, Federal University of São Carlos (UFSCar), São Carlos, SP, Brazil.

3 Department of Gerontology, Federal University of São Carlos (UFSCar), São Carlos, SP, Brazil.

Received: 08/16/2018- Accepted: 04/02/2019

DOl: 10.1590/0101-60830000000197

\begin{abstract}
Background: Mild Cognitive Impairment (MCI) is a disorder in which the patient presents a cognitive decline, but without negative impact on the activities of daily living. Objective: To carry out a systematic review of published studies that analyzed the prevalence of Mild Cognitive Impairment (MCI) in older adults living in the community, and the criteria used for the diagnosis of this disorder. Methods: A search was carried out in May 2017 using the descriptors: "epidemiology" or "prevalence", "mild cognitive impairment", and "community" in the PubMed, PsycInfo, SciELO, Web of Science, and Scopus databases. Two independent researchers extracted and documented the data. We used a random effect model to calculate pooled prevalence of MCI for overall studies and for each subgroup divided by diagnostic criteria. Results: We found initially 1996 articles, and we selected 35 studies. The prevalence of MCI in the selected studies ranged from $0.5 \%$ to $41.8 \%$. The overall pooled prevalence of MCI was $17.3 \%$ (CI 95\%, 13.8-20.8), with significant heterogeneity between estimates (I 2 $=99.6 \%)$. Discussion: The standardization of the diagnostic criteria for MCI, as well as the tests used in the cognitive evaluation, could allow the comparison between the studies and would be an important step in the researches of this area.
\end{abstract}

Pessoa RMP et al. / Arch Clin Psychiatry. 2019;46(3):72-9

Keywords: Prevalence, epidemiology, community, mild cognitive impairment.

\section{Introduction}

Mild cognitive impairment (MCI) is the term used for the disorder between cognitive age changes and early stages of dementia. Patients with this morbidity show a decline in cognitive function with respect to their baseline pattern and has no negative impact on the activities of daily living. The aim of the diagnosis of MCI is to identify individuals at the onset of cognitive decline, although not all individuals will progress to dementia ${ }^{1,2}$.

MCI has received several denominations and definitions over the last 20 years, with Mayo Clinic criteria being the most accepted. The original Mayo MCI criteria evidenced memory impairment with preservation of the other cognitive domains. The criteria include memory loss, preferably corroborated by an informant; target memory impairment; general cognition preserved; preserved activities of daily living; and absence of dementia ${ }^{2}$. Later, research has expanded MCI symptomatology into other cognitive domains, and has considered memory impairment a condition not necessarily present, with the remaining criteria being better known as the Petersen Criteria ${ }^{3}$. Petersen criteria evidenced cognitive complaint, preferably corroborated by an informant; target cognitive impairment; general cognition preserved; preserved activities of daily living; and absence of dementia.

We have studies indicating a conversion of $10 \%$ to $15 \%$ per year of amnestic MCI in AD, for others MCI subtypes the conversion is still undefined, which highlights the importance of this disorder, since dementia is one of the diseases that most overburden developed countries and their health systems. Therefore, preventive measures are urgently needed $4-6$.
Despite the growing importance of MCI, studies in the area are still scarce, being a large part of them performed in clinical settings, such as reference centers for cognitive disorders, generating several implications. For example, depending on the admission mechanism of the research site, some selection criteria applied can recruit only individuals with etiology for this disorder, usually those with a degenerative origin, or with a positive family history for dementia. On the other hand, community-based studies, by definition, would not restrict the nature of the sample, but rather provide heterogeneity ${ }^{2,7}$.

Therefore, the main objective of this study is to carry out a systematic review of all published studies that analyzed the prevalence of MCI in older adults living in the community, and the criteria used for the diagnosis of this disorder.

\section{Methods}

A systematic review of national and international literature, regardless of the date of publication, on the prevalence of mild cognitive impairment in communities was carried out between May 2 and 16, 2017. For this purpose, the following keywords were used: epidemiology; prevalence; mild cognitive impairment; and community. The databases used were PubMed, PsycInfo, SciELO, Web of Science, and Scopus.

Inclusion criteria were studies with: a sample aged over 60 years, performed in communities; publication in English, Portuguese and Spanish; description of criteria used for diagnosis of MCI, and of the prevalence of MCI in the results. 
We excluded studies with specific clinical samples (stroke, acute myocardial infarction, etc.), which used only screening cognitive scales such as Mini-mental Status examination (MMSE), Montreal Cognitive Assessment (MoCA) or Addenbrooke's Cognitive Examination-Revised (ACE-R) to determine MCI. We also excluded letters to the editor, book chapters and reviews, collection of abstracts, comments, notes, errata, theses, dissertations, and bibliographic/ systematic reviews. No time limitation was adopted.

Two independent researchers extracted and documented the following data: authorship; date, year and country of publication; study design; sample size; gender, age and schooling of participants; criteria used for diagnosis and prevalence of MCI. The data were reviewed, and any disagreement was discussed among the authors.

We evaluated the individual quality of the articles using a tool for cross-sectional studies ${ }^{8}$. The tool is composed of ten items that evaluate the external validity, selection, and domain of response bias, internal validity, measurement bias, and analysis. In the end, the study may be classified as low risk (score 0-3), moderate risk (4-6), or high risk (7-9). Of the 35 articles selected, five presented a moderate risk of bias ${ }^{9-13}$. All other articles scored between 0 and 3 (low risk).

We used a random effect model to calculate pooled prevalence of MCI for overall studies and for each subgroup divided by diagnostic criteria. We divided the diagnostic criteria into three subgroups: memory complaint plus decline in cognitive test; cognitive complaint and decline in cognitive test; and decline in cognitive test. The percentage of total variation due to heterogeneity within the subgroups and the overall was evaluated by $\mathrm{I}^{2}$ measure. We also presented the prevalence with 95\% exact confidence intervals for each study, subgroup, and overall studies. We used the Stata statistical software version 14.1 (metaprop_one command) to perform the meta-analyses.

\section{Results}

A total of 1996 articles were found. After exclusion of duplicated articles, 783 articles remained. The abstracts of these articles were reviewed, after which 748 were excluded. The final sample consisted of 35 articles. The review flowchart is shown in Figure 1.
The prevalence of MCI in the selected studies ranged from $0.5 \%^{14}$ to $41.8 \%{ }^{15}$. The overall pooled prevalence of MCI was $17.3 \%$ (CI 95\%, 13.8-20.8), with significant heterogeneity between estimates $\left(\mathrm{I}^{2}=99.6 \%\right)$.

Of the included studies, $40 \%$ were published between 2000 and 2010 , and $60 \%$ after 2010 . Regarding the study design, 19 were cross-sectional and 16 were longitudinal studies. The sample size ranged from 42 participants in a study in Australia ${ }^{11}$ to 10276 participants in a study in China ${ }^{16}$. The country with the highest number of studies was the United States, followed by China.

We divided the studies into three subgroups according to the diagnostic criteria: memory complaint plus decline in cognitive test; cognitive complaint and decline in cognitive test; and decline in cognitive test. We divided the Tables 1, 2 and 3 summarized the selected articles.

The studies differed with respect to cut-off points used in cognitive tests. For example, impairment in cognitive domains, depending on the study, was defined as a performance on tests below 1.5 standard deviation or below 1 standard deviation or below the 10th percentile relative to a reference group or below normative references.

When stratified by diagnostic criteria, the prevalence varied according to the criteria used. The studies using the memory complaint plus cognitive decline had prevalence of $15.0 \%$ (95\% CI $10.4-19.7 \%$; ranging from $0.5 \%$ to $32.6 \%$ ). The studies have used only cognitive tests criteria had higher prevalence $(21.6 \%$; $95 \%$ CI $16.5-26.6 \%)$ than those used cognitive and memory complaints plus decline in cognitive tests (Figure 2). There was significant heterogeneity between prevalence estimates in all subgroups.

We also performed meta-analysis considering the following aspects: sample size $(0-500 ; 501-1000 ;>1000)$, country per capita income and risk of bias. The heterogeneity of the studies remained high $(>75 \%)$ in all subgroups analyzed.

In Figure 3, we presented a graph with the distribution of the studies considering the prevalence and the standard error (funnel plot). We can observe an asymmetric distribution of the studies, suggesting the presence of biases and/or even the heterogeneity of the studies. The publication bias is one of possible causes of asymmetry.

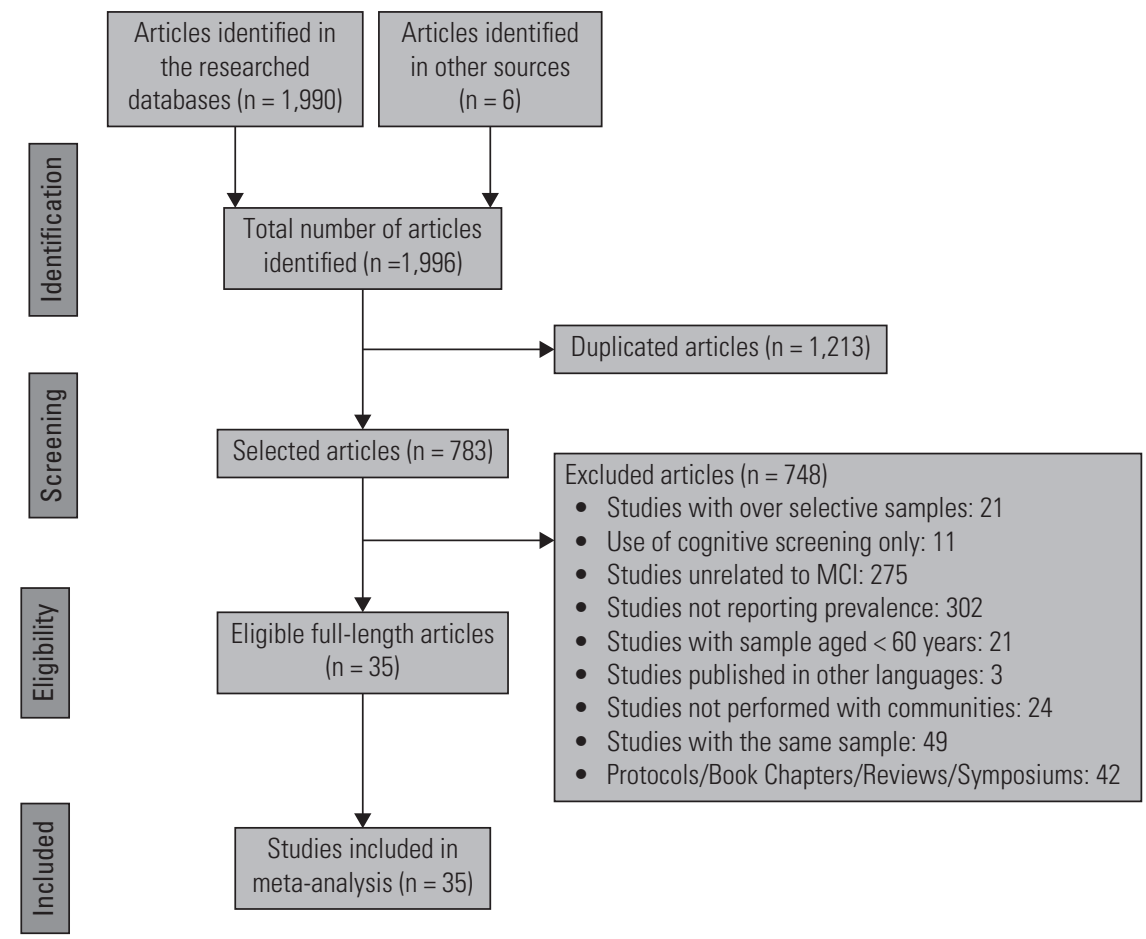

Figure 1. PRISMA flowchart. Excerpted from: Moher D, Liberati A, Tetzlaff J, Altman DG. The PRISMA Group. Preferred Reporting Items for Systematic Reviews and Meta-Analyses: The PRISMA Statement. PLoS Med. 2009;6(6):e1000097. 
Table 1. Characteristics of the selected studies that used cognitive complaint plus decline in cognitive tests as diagnostic criteria

\begin{tabular}{|c|c|c|c|c|c|c|c|c|c|}
\hline Authors & $\begin{array}{l}\text { Country/GNI per } \\
\text { capita }\end{array}$ & Year & Design & Sample & Sex (M) & Age & Schooling & Criteria & Prevalence \\
\hline $\begin{array}{l}\text { Afgin et al. } \\
(2012)^{17}\end{array}$ & $\begin{array}{l}\text { Israel } \\
\text { (High) }\end{array}$ & \begin{tabular}{|l} 
Jan. 2003- \\
Dec. 2008
\end{tabular} & Cohort & \begin{tabular}{|l|}
944 \\
(Total: 1,003)
\end{tabular} & $49.40 \%$ & $\begin{array}{l}\text { MCl: } 72.8( \pm 6.1) \\
\text { Healthy: } \\
70.7( \pm 5.5)\end{array}$ & $\begin{array}{l}3 \text { (3) } \\
51 \% \text { illiterate }\end{array}$ & CDR 0.5 & \begin{tabular}{|l}
$32.1 \%$ \\
(303 subjects)
\end{tabular} \\
\hline $\begin{array}{l}\text { Artero et al. } \\
(2008)^{15}\end{array}$ & $\begin{array}{l}\text { France } \\
\text { (High) }\end{array}$ & 1991-2001 & Cohort & $\begin{array}{l}\text { 6,892 } \\
\text { (Total: 9,313) }\end{array}$ & $\begin{array}{l}\text { MCl: } 35 \% \\
\text { Healthy: } \\
43.4 \%\end{array}$ & $\begin{array}{l}\text { MCl: } 74.6( \pm 5.7) \\
\text { Healthy: } 73.1( \pm 4.9)\end{array}$ & $\begin{array}{l}\text { Low schooling: } \\
\text { MCl: } 24.7 \% \\
\text { Healthy: } 22.5 \%\end{array}$ & $\begin{array}{l}\text { Lowest } \\
\text { quartile }\end{array}$ & \begin{tabular}{|l|}
$42 \%$ \\
(2882 subjects)
\end{tabular} \\
\hline $\begin{array}{l}\text { Ding et al. } \\
(2015)^{18}\end{array}$ & $\begin{array}{l}\text { China } \\
\text { Shanghai Aging } \\
\text { Study } \\
\text { (Middle) }\end{array}$ & $\begin{array}{l}\text { Jan. } 2010- \\
\text { Sep. } 2011\end{array}$ & $\begin{array}{l}\text { Cross- } \\
\text { sectional }\end{array}$ & \begin{tabular}{|l|}
3,141 \\
(Total: 4,519)
\end{tabular} & $\begin{array}{l}46 \% \\
\text { MCl: } 44 \%\end{array}$ & $72.3( \pm 8.1)$ & $\begin{array}{l}\text { Illiterate: } \\
\text { Total: } 4.26 \% \\
\text { MCl: } 8.48 \%\end{array}$ & $1.5 \mathrm{SD}$ & $\begin{array}{l}19.1 \% \\
\text { (601 subjects) } \\
\text { aMCl: 12.5\% (393 } \\
\text { subjects) } \\
\text { na-MCl: } 6.6 \% \text { (208 } \\
\text { subjects) }\end{array}$ \\
\hline $\begin{array}{l}\text { Gao et al. } \\
(2014)^{9}\end{array}$ & $\begin{array}{l}\text { USA } \\
\text { Indianapolis- } \\
\text { Ibadan } \\
\text { DementiaProjetc. } \\
\text { (High) } \\
\end{array}$ & \begin{tabular}{|l|}
$1992-2009$ \\
(Seven \\
assessments)
\end{tabular} & Cohort & $\begin{array}{l}2,212 \\
\text { (Baseline: } \\
1,992)\end{array}$ & \begin{tabular}{|l|}
$35 \%$ \\
Healthy: $36 \%$ \\
MCl: $31.8 \%$
\end{tabular} & $\begin{array}{l}74.3( \pm 6.9) \\
\text { MCI: } 75.3( \pm 7.1) \\
\text { Healthy: } 73.9( \pm 6.8)\end{array}$ & $\begin{array}{l}\text { Total: } \\
9.7( \pm 3.1) \\
\text { MCl: } \\
8.9( \pm 3.1) \\
\text { Healthy: } 9.9( \pm 3) \\
\end{array}$ & $1.5 \mathrm{SD}$ & $\begin{array}{l}14.8 \% \\
\text { (327 subjects) } \\
\text { Year } 1992\end{array}$ \\
\hline $\begin{array}{l}\text { Lee et al. } \\
(2009)^{19}\end{array}$ & $\begin{array}{l}\text { Korea } \\
\text { GDEMCIS } \\
\text { (High) }\end{array}$ & $\begin{array}{l}\text { 1rst phase: } \\
\text { Oct. } \\
\text { 2005-March } \\
2007\end{array}$ & Cohort & $\begin{array}{l}927 \\
\text { (Total: 5,085) } \\
\\
\end{array}$ & \begin{tabular}{l|}
$33.7 \%$ \\
Healthy: \\
$34.5 \%$ \\
MCI1: $48.9 \%$ \\
MCI2: $18.1 \%$ \\
MCI3: $25.9 \%$
\end{tabular} & $\begin{array}{l}72.9( \pm 6.9) \\
\text { Healthy: } 71.05 \\
( \pm 6.02) \\
\text { MCl1: } 69.96( \pm 5.42) \\
\text { MCl2: } 73.63( \pm 6.31) \\
\text { MCl3: } 76.33( \pm 7.12)\end{array}$ & $\begin{array}{l}5.2 \text { Healthy: } \\
6.37( \pm 4.87) \\
\text { MCI1: } 7.26 \\
( \pm 4.39) \text { MCI2: } \\
2.57( \pm 3.17) \\
\text { MCI3: } 3.24 \\
( \pm 4.41)\end{array}$ & Cuttoffs & $\begin{array}{l}7.6 \% \\
\text { (all types of MCI) } \\
\text { (384 subjects) }\end{array}$ \\
\hline $\begin{array}{l}\text { Ogunniyi } \\
\text { et al. } \\
(2016)^{20}\end{array}$ & Nigeria (Middle) & $\begin{array}{l}\text { May-Oct. } \\
2013 \\
\text { Jan.-Feb. } \\
2014\end{array}$ & Cohort & $\begin{array}{l}613 \text { (Total: } \\
642 \text { ) }\end{array}$ & $\begin{array}{l}30.3 \% \\
\text { Healthy: } \\
31.7 \% \mathrm{MCl}: \\
19.8 \%\end{array}$ & $72.9( \pm 8.5)$ & $\begin{array}{l}\text { Literate: } \\
\text { Healthy: 33.6\% } \\
\text { MCl 13.5\% }\end{array}$ & Cuttoffs & $\begin{array}{l}18.1 \% \\
\text { (111 subjects) } \\
\text { SDa-MCI: } 42.3 \% \\
\text { MDa-MCI: } 40.5 \% \\
\text { SDna-MCI: } 16.2 \% \\
\text { MDna-MCI: } 0.9 \% \\
\end{array}$ \\
\hline $\begin{array}{l}\text { Olazarán } \\
\text { et al. } \\
(2015)^{12}\end{array}$ & $\begin{array}{l}\text { Spain } \\
\text { The Vallecas } \\
\text { Project } \\
\text { (High) }\end{array}$ & $\begin{array}{l}\text { Oct. 2011- } \\
\text { Dec. } 2013\end{array}$ & Cohort & \begin{tabular}{|l|}
1,169 \\
(Total: 2,077)
\end{tabular} & $36.5 \%$ & Total: $74.4( \pm 3.9)$ & $\begin{array}{l}\text { < Primary school } \\
\text { Total: } 18.6 \%\end{array}$ & $1.5 \mathrm{SD}$ & $\begin{array}{l}\text { MCl: } 7 \% \\
\text { (82 subjects) } \\
\text { aMCl: } 3.1 \% \\
\text { naMCl: } 0.1 \% \\
\text { mixed } \mathrm{MCl}: 3.8 \% \\
\end{array}$ \\
\hline $\begin{array}{l}\text { Petersen } \\
\text { et al. } \\
(2010)^{21}\end{array}$ & $\begin{array}{l}\text { USA } \\
\text { The Mayo Clinic } \\
\text { Study of Ageing } \\
\text { (High) }\end{array}$ & $\begin{array}{l}\text { Oct. 2004- } \\
\text { July } 2007\end{array}$ & Cohort & \begin{tabular}{|l|}
2,050 \\
(Total: 4,398)
\end{tabular} & MCl: 58.35\% & - & - & 1.0 SD & $\begin{array}{l}16 \% \\
\text { (329 subjects) } \\
\text { SDa-MCl: } 11.6 \% \\
\text { MDa-MCl: } 4.5 \% \\
\text { SDna-MCI: } 3.4 \% \\
\text { MDna-MCl: } 1.1 \%\end{array}$ \\
\hline $\begin{array}{l}\text { Pilleron } \\
\text { et al. } \\
(2015)^{22}\end{array}$ & $\begin{array}{l}\text { Central Africa } \\
\text { CAR: Central } \\
\text { African Republic } \\
\text { ROC: Republic of } \\
\text { the Congo } \\
\text { (Low) } \\
\end{array}$ & \begin{tabular}{|l} 
Nov. 2011- \\
Dec. 2012
\end{tabular} & \begin{tabular}{|l|} 
Cross- \\
sectional
\end{tabular} & $\begin{array}{l}2,002 \\
\text { CAR: } 973 \\
\text { ROC: } 1029\end{array}$ & $\begin{array}{l}\text { CAR: } 37.9 \% \\
\text { ROC: } 39.2 \%\end{array}$ & $\begin{array}{l}\text { CAR: } 72.7( \pm 6.5) \\
\text { ROC: } 73.8( \pm 6.9)\end{array}$ & $\begin{array}{l}\text { Illiterate: Total: } \\
\text { CAR: } 69.2 \% \\
\text { ROC: } 68.4 \%\end{array}$ & Cuttoffs & \begin{tabular}{|l|}
$6,6 \%$ \\
(133 subjects) \\
7.2 (CAR) \\
(70 subjects) \\
$6.1 \%$ (ROC) \\
(63 subjects) \\
\end{tabular} \\
\hline $\begin{array}{l}\text { Richard } \\
\text { et al. } \\
(2013)^{23}\end{array}$ & $\begin{array}{l}\text { USA } \\
\text { WHICAP } \\
\text { (High) }\end{array}$ & 1999-2001 & Cohort & \begin{tabular}{|l|}
2,160 \\
(Total: 2,183)
\end{tabular} & - & - & - & - & $\begin{array}{l}19.86 \% \\
\text { (429 subjects) } \\
51.7 \% \text { aMCl } \\
48.3 \% \text { naMCl } \\
\end{array}$ \\
\hline $\begin{array}{l}\text { Tiwari et al. } \\
(2013)^{24}\end{array}$ & \begin{tabular}{|l|l|} 
India \\
(Middle) \\
\end{tabular} & $2008-2010$ & $\begin{array}{l}\begin{array}{l}\text { Cross- } \\
\text { sectional }\end{array} \\
\end{array}$ & \begin{tabular}{|l|}
2,146 \\
(Total: 2,324$)$ \\
\end{tabular} & Total: 47.4\% & Total: $67.8( \pm 5.9)$ & - & - & \begin{tabular}{|l|}
$4.6 \%$ \\
(99 subjects)
\end{tabular} \\
\hline $\begin{array}{l}\text { Vanoh et al. } \\
(2017)^{25}\end{array}$ & $\begin{array}{l}\text { Malaysia } \\
\text { TUA } \\
\text { (Middle) }\end{array}$ & 4 years long & Longitudinal & 1,993 & $\begin{array}{l}\text { Total: } 50.3 \% \\
\text { MCl: } 56.5 \%\end{array}$ & $\begin{array}{l}\text { Total: } 68.51( \pm 5.93) \\
\text { MCl: } 69.45( \pm 5.98)\end{array}$ & $\begin{array}{l}\text { Total: } \\
5.54( \pm 3.94) \\
\text { MCl: } \\
4.62( \pm 3.18) \\
\end{array}$ & $1.5 \mathrm{SD}$ & $\begin{array}{l}16 \% \\
\text { (315 subjects) }\end{array}$ \\
\hline $\begin{array}{l}\text { Yu et al. } \\
(2016)^{26}\end{array}$ & \begin{tabular}{|l} 
China \\
(Middle)
\end{tabular} & - & Cohort & \begin{tabular}{|l|}
376 \\
(Total: 480)
\end{tabular} & - & $\begin{array}{l}\text { Healthy: } 68.3( \pm 4.1) \\
\text { MCl: } 68.6( \pm 4.7)\end{array}$ & $\begin{array}{l}\text { Healthy: } \\
8 \text { ( } \pm 4.3) \\
\text { MCl: } \\
7.1( \pm 4.2)\end{array}$ & - & $\begin{array}{l}17.6 \% \\
\text { (66 subjects) }\end{array}$ \\
\hline
\end{tabular}

GNI: Gross National income; MCl: mild cognitive impairment; CDR: clinical dementia rating; SD: standard deviation; aMCl: amnestic mild cognitive impairment; naMCl: nonamnestic mild cognitive impairment; SDa-MCl: single-domain amnestic MCl; MDa-MCl: multiple-domain amnestic MCl; SDna-MCl: single-domain non-amnestic MCl; MDna-MCI: multiple-domain amnestic. 
Table 2. Characteristics of the selected studies that used memory complaint plus decline in cognitive tests as diagnostic criteria

\begin{tabular}{|c|c|c|c|c|c|c|c|c|c|}
\hline Authors & $\begin{array}{l}\text { Country/ } \\
\text { GNI per } \\
\text { capita }\end{array}$ & Year & Design & Sample & Sex (M) & Age & Schooling & Criteria & Prevalence \\
\hline $\begin{array}{l}\text { Choi et al. } \\
(2008)^{27}\end{array}$ & $\begin{array}{l}\text { Korea } \\
\text { (High) }\end{array}$ & $\begin{array}{l}\text { July 2005- } \\
\text { Feb. } 2007\end{array}$ & \begin{tabular}{|l|} 
Cross- \\
sectional
\end{tabular} & $\begin{array}{l}\text { 175 (Total: } \\
1,215)\end{array}$ & $\begin{array}{l}\text { 53.2\% Healthy: } 2 \% \\
\text { MCl: } 59.6 \%\end{array}$ & $\begin{array}{l}\text { Total: } 74.3( \pm 16.7) \\
\text { MCl: } 73.82( \pm 4.51) \\
\text { Healthy: } 71.88 \\
( \pm 4.17)\end{array}$ & $\begin{array}{l}\text { MCl: } \\
4.93( \pm 3.27) \\
\text { Healthy: } 5.85 \\
( \pm 4.63)\end{array}$ & CDR 0.5 & $\begin{array}{l}32.9 \% \\
\text { (57 subjects) }\end{array}$ \\
\hline $\begin{array}{l}\text { Juarez- } \\
\text { Cedillo et } \\
\text { al. (2012)28 }\end{array}$ & $\begin{array}{l}\text { Mexico } \\
\text { SADEM } \\
\text { (Middle) }\end{array}$ & $\begin{array}{l}\text { Sep. } \\
\text { 2009-March } \\
2010\end{array}$ & $\begin{array}{l}\text { Cross- } \\
\text { sectional }\end{array}$ & $\begin{array}{l}3,036 \\
\text { (Total: } \\
3,191)\end{array}$ & $\begin{array}{l}\text { 42\% MCl: } 36.3 \% \\
\text { Healthy: } 42.6 \%\end{array}$ & $\begin{array}{l}71.2( \pm 7.5) \\
\text { MCl: } 75.3( \pm 7.9) \\
\text { Healthy: } \\
70.9( \pm 7.3)\end{array}$ & $\begin{array}{l}\text { Total: } 6.1( \pm 4.6) \\
\text { MCl: } 5.4( \pm 5.5) \\
\text { Healthy: } 6.2 \\
( \pm 4.6)\end{array}$ & $1.5 \mathrm{SD}$ & $\begin{array}{l}\text { 6.25\% } \\
\text { (190 subjects) } \\
\text { SDa-MCl: } 2.41 \% \\
\text { MDa-MCl: } 2.56 \% \\
\text { SDna-MCl: } 1.18 \% \\
\text { MDna-MCl: } 0.3 \% \\
\end{array}$ \\
\hline $\begin{array}{l}\text { Jungwirth } \\
\text { et al. } \\
(2005)^{14}\end{array}$ & $\begin{array}{l}\text { Austria } \\
\text { VITA } \\
\text { (High) }\end{array}$ & $\begin{array}{l}\text { Start in May } \\
2000\end{array}$ & \begin{tabular}{|l|} 
Cross- \\
sectional
\end{tabular} & $\begin{array}{l}592 \text { (Total: } \\
1,505 \text { ) }\end{array}$ & $\begin{array}{l}\text { Memory only: } 50 \% \\
\text { Only lack of memory: } \\
30.7 \% \\
\text { Memory + lack of } \\
\text { memory: } 25.8 \% \\
\text { Petersen: } 66.7 \%\end{array}$ & $\begin{array}{l}\text { Memory only: } 75.71 \\
( \pm 0.44) \\
\text { Lack of memory } \\
\text { only: } 75.83( \pm 0.46) \\
\text { Memory + lack } \\
\text { of memory: } 75.89 \\
( \pm 0.46) \\
\text { Petersen: } 75.3( \pm 0.2)\end{array}$ & \begin{tabular}{|l|} 
Memory only: \\
$10.5( \pm 1.9)$ \\
Lack of memory \\
only: $9.5( \pm 2)$ \\
Memory + lack \\
of memory: 9.4 \\
$( \pm 1.6)$ \\
Petersen: 9.7 \\
$( \pm 2.1)$
\end{tabular} & $1.5 \mathrm{SD}$ & $\begin{array}{l}\text { Memory only: } 3.7 \% \\
\text { (22 subjects) } \\
\text { Lack of memory only: } \\
14.9 \% \\
\text { (88 Subjects) } \\
\text { Memory + lack of memory: } \\
5.2 \% \\
\text { (31 subjects) } \\
\text { Petersen- } \mathrm{MCl} \text { - amnestic: } \\
0.5 \% \text { (3 subjects) }\end{array}$ \\
\hline $\begin{array}{l}\text { Katz et al. } \\
(2012)^{29}\end{array}$ & $\begin{array}{l}\text { USA } \\
\text { Eisntein } \\
\text { Aging } \\
\text { Study } \\
\text { (High) }\end{array}$ & 1993-2004 & Cohort & 1,944 & $39.3 \%$ & $78.8( \pm 5.42)$ & $\begin{array}{l}\text { Total: } 13.5 \\
( \pm 3.5)\end{array}$ & $1.5 \mathrm{SD}$ & $\begin{array}{l}20 \% \\
\text { (390 subjects) } \\
\text { aMCl: } 11.6 \% \\
\text { (226 subjects) } \\
\text { naMCl: } 9.9 \% \\
\end{array}$ \\
\hline $\begin{array}{l}\text { Khedr et al. } \\
(2015)^{30}\end{array}$ & \begin{tabular}{|l} 
Egypt \\
(Middle)
\end{tabular} & $\begin{array}{l}\text { Sep. 2011- } \\
\text { Aug. } 2013\end{array}$ & \begin{tabular}{|l|}
$\begin{array}{l}\text { Cross- } \\
\text { sectional }\end{array}$ \\
\end{tabular} & 691 & MCl: $58 \%$ & MCl: $67.3( \pm 7.1)$ & $\begin{array}{l}\text { Illiterate: } \mathrm{MCl}: \\
66 \%\end{array}$ & $1.5 \mathrm{SD}$ & 1.74\% (12 subjects) \\
\hline $\begin{array}{l}\text { Kumar et } \\
\text { al. }(2005)^{31}\end{array}$ & $\begin{array}{l}\text { Australia } \\
\text { PATH } 60+ \\
\text { (High) }\end{array}$ & $\begin{array}{l}\text { 1st phase: } \\
\text { 2001/2002 }\end{array}$ & Cohort & $\begin{array}{l}2,518 \\
\text { (Total: } \\
4,378)\end{array}$ & - & - & 13.72 & Cuttoffs & $\begin{array}{l}\text { MCl: } 3.7 \% \\
\text { (93 subjects) } \\
\text { MND: } 0,6 \% \\
\text { (15 subjects) } \\
\end{array}$ \\
\hline $\begin{array}{l}\text { Lee et al. } \\
(2009)^{32}\end{array}$ & $\begin{array}{l}\text { Korea } \\
\text { KLoSHA } \\
\text { (High) }\end{array}$ & 2005 & $\begin{array}{l}\text { Cross- } \\
\text { sectional }\end{array}$ & 714 & $32.2 \%$ & $71.9( \pm 5.7)$ & $\begin{array}{l}<6 \text { years of } \\
\text { schooling: } \\
\text { Total: } 50.7 \%\end{array}$ & $1.5 \mathrm{SD}$ & $\begin{array}{l}\text { SMC: } 27.59 \% \\
\text { (197 subjects) } \\
\text { CDR 0.5: } 17.9 \% \\
\text { (aMCI: } 59.9 \% \text { and naMCl: } \\
40.1 \% \text { ) }\end{array}$ \\
\hline $\begin{array}{l}\text { Lee et al. } \\
(2012)^{33}\end{array}$ & \begin{tabular}{|l} 
Malaysia \\
(Middle)
\end{tabular} & $\begin{array}{l}\text { Dec. 2008- } \\
\text { May } 2009\end{array}$ & \begin{tabular}{|l|} 
Cross- \\
sectional
\end{tabular} & $\begin{array}{l}318 \text { (Total: } \\
\text { 333) }\end{array}$ & $40.9 \%$ & $65.9( \pm 5.3)$ & Total: $5.8( \pm 3.5)$ & $1.5 \mathrm{SD}$ & $\begin{array}{l}\text { MCl: } 21.1 \% \\
\text { (67 subjects) } \\
\text { aMCl: } 15.4 \% \\
\text { (49 subjects) } \\
\text { naMCl: } 5.7 \%\end{array}$ \\
\hline $\begin{array}{l}\text { Louis et al. } \\
(2005)^{34}\end{array}$ & $\begin{array}{l}\text { USA } \\
\text { (High) }\end{array}$ & $\begin{array}{l}\text { 1992/1999- } \\
2001\end{array}$ & Cohort & $\begin{array}{l}\text { 2,230 } \\
\text { (Total: } \\
2,776)\end{array}$ & $\begin{array}{l}32.5 \% \\
\text { Healthy: } 32 \% \\
\text { a-MCl: } 34.5 \% \\
\text { na-MCl: } 33.3 \%\end{array}$ & $\begin{array}{l}77.2( \pm 6.6) \\
\text { Healthy: } 77( \pm 6.6) \\
\text { a-MCl: } 78.1( \pm 7) \\
\text { na-MCI: } 77.1( \pm 6.6)\end{array}$ & $\begin{array}{l}10.3( \pm 4.8) \\
\text { Healthy: } 10.5 \\
\text { ( } \pm 4.7) \text { a-MCl: } \\
10.2 \text { ( } \pm 4.6) \text { na- } \\
\text { MCl: } 9.6( \pm 5)\end{array}$ & $1.5 \mathrm{SD}$ & $\begin{array}{l}27.3 \% \text { (aMCI } 42 \% \text { and } \\
\text { na MCl: } 58 \%) \\
\text { (608 subjects) }\end{array}$ \\
\hline $\begin{array}{l}\text { Ma et al. } \\
(2016)^{35}\end{array}$ & $\begin{array}{l}\text { China } \\
\text { (Middle) }\end{array}$ & $\begin{array}{l}\text { Jan.-May } \\
2012\end{array}$ & $\begin{array}{l}\text { Cross- } \\
\text { sectional }\end{array}$ & $\begin{array}{l}5,214 \\
\text { (Total: } \\
5,291)\end{array}$ & $\begin{array}{l}\text { 43.9\% Healthy: } \\
\text { 42.19\% MCl: } 40.4 \%\end{array}$ & $72.13( \pm 4.22)$ & $6.34( \pm 7.26)$ & $1.5 \mathrm{SD}$ & $\begin{array}{l}11.33 \% \\
\text { (574 subjects) } \\
\text { SDa-MCl: } 4.48 \% \\
\text { (227 subjects) } \\
\text { MDa-MCl: } 2.09 \% \\
\text { (106 subjects) } \\
\text { SDna-MCl: } 4.22 \% \\
\text { (214 subjects) } \\
\text { MDna-MCl: } 0.53 \% \\
\text { (27 subjects) }\end{array}$ \\
\hline $\begin{array}{l}\text { Purser et } \\
\text { al. }(2005)^{36}\end{array}$ & $\begin{array}{l}\text { USA } \\
\text { EPESE } \\
\text { (High) }\end{array}$ & $\begin{array}{l}\text { 1981, 1984, } \\
\text { 1987, } 1991\end{array}$ & Cohort & 3,673 & $39.7 \%$ & 74 & Total: 11 & Cuttoffs & $\begin{array}{l}22 \% \\
\text { (810 subjects) }\end{array}$ \\
\hline $\begin{array}{l}\text { Shimada et } \\
\text { al. }(2013)^{37}\end{array}$ & $\begin{array}{l}\text { Japan } \\
\text { OSHPE } \\
\text { (High) }\end{array}$ & $2011 / 2012$ & $\begin{array}{l}\text { Cross- } \\
\text { sectional }\end{array}$ & $\begin{array}{l}5,025 \\
\text { (Total: } \\
5,104) \\
\end{array}$ & $\begin{array}{l}\text { Healthy: } 39.9 \% \\
\text { MCl: } 48.2 \%\end{array}$ & - & - & 1.5 SD & $18.8 \%$ (945 subjects) \\
\hline $\begin{array}{l}\text { Wang et } \\
\text { al. }(2015)^{38}\end{array}$ & $\begin{array}{l}\text { China } \\
\text { (Middle) }\end{array}$ & \begin{tabular}{|l} 
Jan. 2010- \\
Jan. 2011
\end{tabular} & $\begin{array}{l}\text { Cross- } \\
\text { sectional }\end{array}$ & 3,136 & Total: $40.65 \%$ & Total: 69.3 ( \pm 6.8$)$ & - & $1.5 \mathrm{SD}$ & $20 \%$ (625 subjects) \\
\hline
\end{tabular}

GNI: Gross National income; MCI: mild cognitive impairment; CDR: clinical dementia rating; SD: standard deviation; SDa-MCl: single-domain amnestic MCl; MDa-MCl: multiple-domain amnestic MCl; SDna-MCl: single-domain non-amnestic MCl; MDna-MCl: multiple-domain amnestic; aMCl: amnestic mild cognitive impairment; naMCl: nonamnestic mild cognitive impairment; MND: mild neurocognitive disorder; SMC: subjective memory complaint. 
Table 3. Characteristics of the selected studies that used decline in cognitive tests as diagnostic criteria

\begin{tabular}{|c|c|c|c|c|c|c|c|c|c|}
\hline Authors & $\begin{array}{l}\text { Country/GNI } \\
\text { per capita }\end{array}$ & Year & Design & Sample & $\operatorname{Sex}(M)$ & Age & Schooling & Criteria & Prevalence \\
\hline $\begin{array}{l}\text { César et al. } \\
(2016)^{39}\end{array}$ & $\begin{array}{l}\text { Brazil } \\
\text { (Middle) }\end{array}$ & 2012 & Cross-sectional & $\begin{array}{l}630 \\
\text { (Total: 738) }\end{array}$ & $\begin{array}{l}37 \% \\
\text { Healthy: } 34.3 \% \\
\text { MCl: } 39.3 \%\end{array}$ & $\begin{array}{l}71.28 \text { ( } \pm 7.99) \\
\text { MCl: } 72.44 \\
\text { ( } \pm 7.72) \\
\text { Healthy: } 69.26 \\
( \pm 7.03)\end{array}$ & $\begin{array}{l}\text { Total: } 4.9 \\
\text { ( } \pm 4.54) \\
\text { MCI: } 4.23 \\
\text { ( } \pm 4.16) \\
\text { Healthy: } \\
5.61( \pm 4.83) \\
\end{array}$ & Cuttoffs & $\begin{array}{l}19.5 \% \text { (135 } \\
\text { subjects) }\end{array}$ \\
\hline $\begin{array}{l}\text { Hilal et al. } \\
(2013)^{40}\end{array}$ & $\begin{array}{l}\text { Singapore } \\
\text { EDIS (High) }\end{array}$ & $\begin{array}{l}\text { Aug. 2010-Feb. } \\
2012 .\end{array}$ & Cross-sectional & $\begin{array}{l}1,226 \text { (Total: } \\
1,538)\end{array}$ & - & Total: 68.2 & - & $1.5 \mathrm{SD}$ & \begin{tabular}{|l|}
$13.3 \%$ \\
(164 subjects) \\
Mild MCl: \\
$6.9 \%$ \\
(84 subjects) \\
Moderate $\mathrm{MCl}$ : \\
$6.5 \%$ \\
(80 subjects) \\
\end{tabular} \\
\hline $\begin{array}{l}\text { Hughes et al. } \\
(2012)^{41}\end{array}$ & $\begin{array}{l}\text { USA } \\
\text { MYHAT } \\
\text { (High) }\end{array}$ & 2006-2008 & Cross-sectional & $\begin{array}{l}\text { 1,737 } \\
\text { (Total: 1,982) }\end{array}$ & $\begin{array}{l}37.3 \% \\
\text { Healthy: } 37.7 \% \\
\text { MCl: } 37.6 \%\end{array}$ & $\begin{array}{l}77.23 \text { ( } \pm 7.33) \\
\text { MCl: } 77.72 \\
( \pm 7.38) \\
\text { Healthy: } 76.95 \\
( \pm 7.29)\end{array}$ & $\begin{array}{l}\text { s High school: } \\
\text { Total: } 13.24 \% \\
\text { MCl: } 12.84 \% \\
\text { Healthy: } \\
13.46 \%\end{array}$ & $\begin{array}{l}\text { Normative } \\
\text { reference }\end{array}$ & $\begin{array}{l}35.9 \% \\
\text { (623 subjects) } \\
\text { *78: SDa-MCI } \\
\text { *136: MDa- } \\
\text { MCl *289: } \\
\text { SDna-MCl } \\
\text { *120: MDna- } \\
\text { MCl }\end{array}$ \\
\hline $\begin{array}{l}\text { Jager and } \\
\text { Budge } \\
(2005)^{10}\end{array}$ & $\begin{array}{l}\text { UK } \\
\text { (High) }\end{array}$ & $\begin{array}{l}4 \text { year of } \\
\text { study, with } 3 \\
\text { assessments }\end{array}$ & Cohort & 157 & - & - & - & $1.5 \mathrm{SD}$ & $\begin{array}{l}25.5 \% \mathrm{~T} 1 \\
\text { (40 subjects) }\end{array}$ \\
\hline $\begin{array}{l}\text { Jia et al. } \\
(2013)^{16}\end{array}$ & $\begin{array}{l}\text { China } \\
\text { (Middle) }\end{array}$ & $\begin{array}{l}\text { Oct. 2008-0ct. } \\
2009\end{array}$ & Cross-sectional & $\begin{array}{l}10,276 \\
\text { (59.3\% urban } \\
\text { area and } \\
40.6 \% \text { rural } \\
\text { area) } \\
\text { (Total: } 13,806)\end{array}$ & $\begin{array}{l}\text { (Urban: } 43.2 \% \\
\text { Rural: } 41.8 \% \text { ) }\end{array}$ & - & $\begin{array}{l}\text { Illiterate: } \\
\text { Total: } \\
\text { (Urban: 17.7\% } \\
\text { Rural: 48.2\%) }\end{array}$ & $1.5 \mathrm{SD}$ & \begin{tabular}{|l|}
$20.8 \%$ \\
(2137 subjects) \\
Urban: $17 \%$ \\
Rural: $25.1 \%$
\end{tabular} \\
\hline $\begin{array}{l}\text { Low et al. } \\
(2004)^{11}\end{array}$ & $\begin{array}{l}\text { Australia } \\
\text { (High) }\end{array}$ & - & Cross-sectional & 42 (Total: 127) & $\begin{array}{l}59.5 \% \\
\text { Healthy: } 63 \% \\
\text { MCI: } 50 \%\end{array}$ & $\begin{array}{l}74.38( \pm 2.47) \\
\text { Healthy: } 73.3 \\
( \pm 2.4) \\
\text { MCl: } 75.5 \\
( \pm 2.2) \\
\end{array}$ & $\begin{array}{l}11.26( \pm 3.2) \\
\text { Healthy: } 11.8 \\
\text { ( } \pm 3.1) \text { MCl: } \\
10.3( \pm 3.4)\end{array}$ & $1.5 \mathrm{SD}$ & $\begin{array}{l}11 \% \mathrm{MCl} \\
\text { (14 subjects) } \\
3.14 \% \text { aMCl } \\
\text { (4 subjects) }\end{array}$ \\
\hline $\begin{array}{l}\text { Paddick et al. } \\
(2015)^{42}\end{array}$ & $\begin{array}{l}\text { Tanzania } \\
\text { (Middle) }\end{array}$ & April-Sep. 2009 & Cross-sectional & 296 & MCl: $21.7 \%$ & MCl: 82 & & $\begin{array}{l}\text { Normative } \\
\text { reference }\end{array}$ & \begin{tabular}{|l}
$15.5 \%$ \\
(46 subjects)
\end{tabular} \\
\hline $\begin{array}{l}\text { Saxton et al. } \\
(2009)_{43}\end{array}$ & $\begin{array}{l}\text { USA } \\
\text { GEM } \\
\text { (High) }\end{array}$ & $\begin{array}{l}\text { Assessments } \\
\text { every } 6 \text { months }\end{array}$ & Cohort & 3,063 & $\begin{array}{l}53.8 \% \\
\text { Healthy: } 52.4 \% \\
\text { CDR: } 57.8 \% \\
\text { NP: } 54.9 \% \\
\text { CDR+NP: } \\
50.6 \%\end{array}$ & $\begin{array}{l}78.5( \pm 3.3) \\
\text { Healthy: } 78 \\
( \pm 2.9) \\
\text { CDR: } 78.7 \\
( \pm 3.4) \\
\text { NP: } 78.7( \pm 3.5) \\
\text { CDR+NP: } 79.6 \\
( \pm 3.7)\end{array}$ & $\begin{array}{l}\text { Healthy: } \\
14.5( \pm 2.8) \\
\text { CDR: } \\
13.8( \pm 2.8) \\
\text { NP: } 15( \pm 3.1)\end{array}$ & $1.5 \mathrm{SD}$ & $\begin{array}{l}\text { CDR: } 40.2 \% \\
\text { (1232 subjects) } \\
\text { NP: } 28.1 \% \\
\text { (861 subjects) } \\
\text { CDR+NP: } \\
15.7 \% \\
\text { (480 subjects) }\end{array}$ \\
\hline $\begin{array}{l}\text { Trittschuh et } \\
\text { al. }(2011)^{44}\end{array}$ & $\begin{array}{l}\text { USA } \\
\text { ACT } \\
\text { (High) }\end{array}$ & 2007 & Cross-sectional & 159 (Total: 200) & $40.9 \%$ & $80.2( \pm 6.5)$ & $15.4( \pm 3.2)$ & $\begin{array}{l}\text { Factor 1: } \\
\text { standard vs. } \\
\text { individual cut- } \\
\text { off points } \\
\text { Factor 2: } \\
\text { Severity of the } \\
\text { impairment } \\
\text { (1vs1.5) } \\
\text { Factor 3: one } \\
\text { vs. mean. }\end{array}$ & $\begin{array}{l}\text { 11.3\%-91.8\% } \\
\text { SDa-MCl: } \\
\text { 2.5-10.7\% } \\
\text { MDa-MCl: } \\
\text { 1.9-61\% }\end{array}$ \\
\hline
\end{tabular}

GNI: Gross National income; MCl: mild cognitive impairment; CDR: clinical dementia rating; SD: standard deviation; SDa-MCl: single-domain amnestic MCl; MDa-MCl: multiple-domain amnestic MCl; SDna-MCl: single-domain non-amnestic MCl; MDna-MCl: multiple-domain amnestic; aMCl: amnestic mild cognitive impairment; NP: neuropsychological tests. 


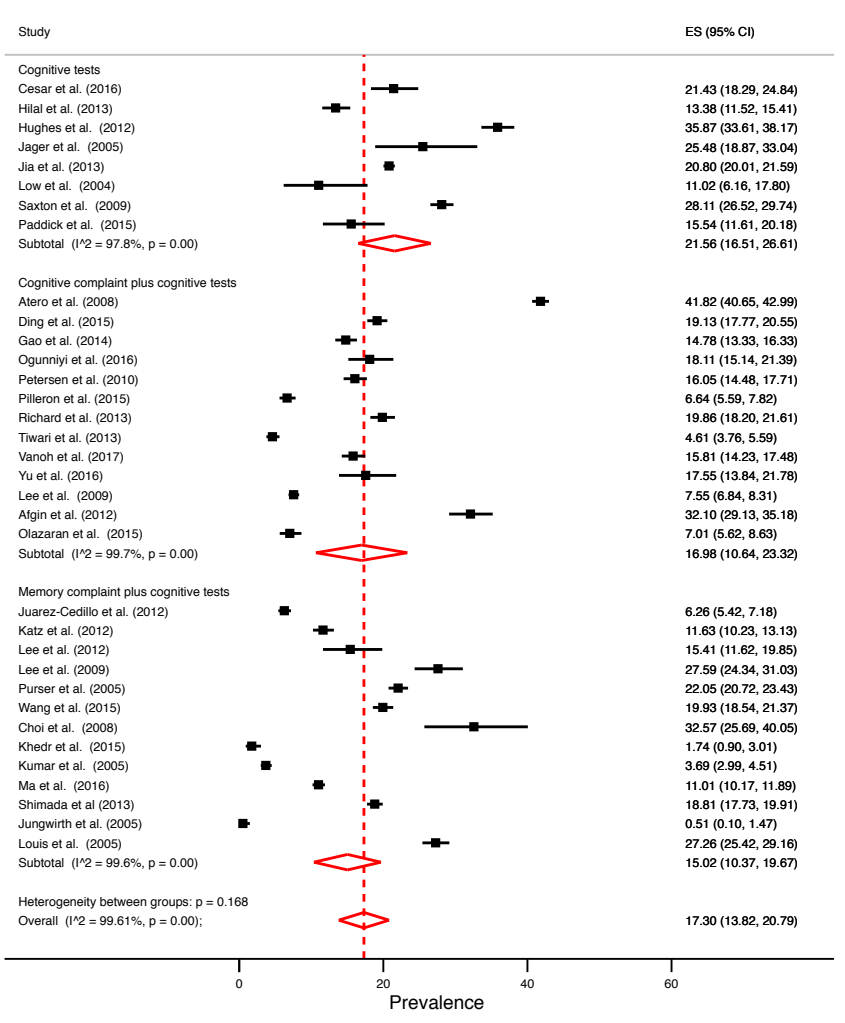

Figure 2. Forest plot for prevalence of mild cognitive impairment in the community stratified by diagnostic criteria.

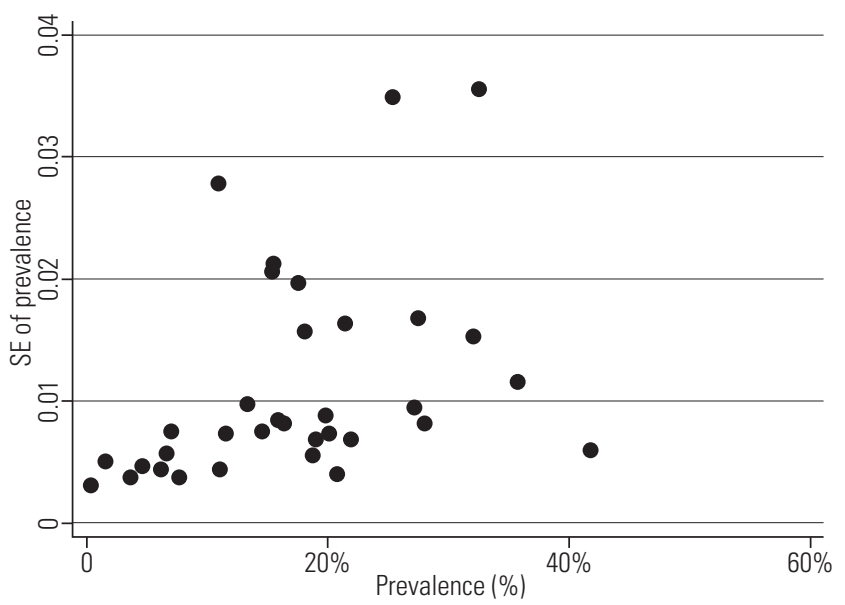

Figure 3. Distribution of the studies considering the prevalence and the standard error (funnel plot).

\section{Discussion}

The present study showed that prevalence rates of MCI in the community can have a large variation. Differences in the characteristics of the studies, such as mean age and schooling of the sample, diagnostic criteria, cognitive tests used, and operationalization of these criteria, may account for this variability.

The studies, even when divided according to the same diagnostic criteria used for MCI, presented high heterogeneity, precluding a valid prevalence. These results can vary considerably from each other, because of the sample definition and how the procedures are performed. This fact hinders a comparison between the studies, emphasizing the need for standardized criteria.
Despite the heterogeneity found, an important feature of the present review was the use of community-based studies alone. In this type of study, shorter assessments with screening tests that can generate erroneous data are usually chosen. A refined clinical evaluation is often needed for MCI diagnosis ${ }^{2}$. Another aspect to be taken into account is that voluntary participation is more significant in community studies than those conducted in academic settings, such as hospitals or clinics, what facilitates the adherence of participants. Especially in the case of more advanced ages, which correspond to the MCI profile, patients who refuse to participate in studies likely have stronger cognitive impairment than the participants. ${ }^{45}$. Another limitation of the study was the non-use of grey literature, which could generate a publication bias.

Regarding the method for diagnosis of MCI, when only cut-off points in neuropsychological assessment were used to determine prevalence rates, without a clinical evaluation, higher values were found, such as the method used in the study of Trittschuch et al. (2016), that reported a prevalence of $91.8 \%$. This study tested different cut-off points for neuropsychological tools, like standard versus individualized, severity of impairment (1.0 versus $1.5 \mathrm{SD}$ ), and level of impairment (any versus average).

Diagnosis based only on neuropsychological assessment does not consider essential criteria for MCI as clinical complaints and the absence of loss of functionality. It is important to emphasize that, although scales are useful in certain environments, they have several limitations and should not be equated with clinical criteria ${ }^{2}$.

The concomitant use of clinical evaluation and neuropsychological status resulted in lower MCI prevalence rates compared to the studies that used only decline in cognitive tests. However, the criterion of subjective cognitive complaints may be disadvantageous in studies not performed in selective samples of memory clinics, since a large percentage of individuals with memory impairments do not express complaints related to this and cognitive complaints may be associated with other pathologies, such as anxiety and depressive disorders ${ }^{46}$. On the other hand, subjective cognitive complaints are usually the only sign of incipient cognitive deterioration in people with high schooling who do not show impairment in cognitive performance if a degree of dementia occur in people with higher schooling, these situations are more advanced and associated with a faster cognitive decline than in people with low schooling ${ }^{5,47}$.

Only two studies used the Clinical Dementia Rating (CDR) value of 0.5 along with medical history as diagnostic criteria. CDR is a scale that ranges from normal (CDR 0) to questionable dementia (CDR 0.5 ) and then to various stages of dementia, mild (CDR 1), moderate (CDR 2) and severe (CDR 3). Some studies consider CDR 0.5 as MCI; nevertheless, it is important to note that CDR is not a diagnostic tool, but rather a severity scale. Therefore, individuals with CDR 0.5 can meet both MCI and mild dementia criteria ${ }^{2}$.

Therefore, it is advisable that cognitive decline be assessed through various and objective tests rather than a single subjective or punctual objective assessment ${ }^{45}$. Furthermore, since the course of changes in normal individuals is variable and tool-dependent a good diagnostic anamnesis, especially in the case of pathologies such as $\mathrm{MCI}$, is necessary along with screening tests ${ }^{46}$.

This review also highlights the importance of standardization for operationalization of MCI criteria, such as in what concerns objective cognitive impairment. Core clinical criteria for MCI (due to Alzheimer Disease) include cognitive impairment in one or more domains compared to appropriate normative data with a suggested deficit level of 1.0-1.5 SD below normative expectations ${ }^{48}$. If this is defined as a performance below 1.5 standard deviations below the mean of the reference group, the prevalence of MCI, from pure statistical reasons, will be lower than if the criterion is performance below 1 standard deviation. Moreover, studies with a short cognitive battery may fail to diagnose cases of lighter MCI due to low sensitivity, leading to false-negative cases 5 .

Although current most used criteria provide guidance on an operational definition of cognitive impairment in MCI, the literature reveals great variability in how MCI has been defined. 
Slight alterations to the operational criteria for neuropsychological impairment in MCI can result in anywhere from 10 to $74 \%$ of samples being identified as MCI ${ }^{49}$.

The criteria for MCI diagnosis should be better standardized, as well as their operation, to facilitate the comparability of different epidemiological and clinical findings. This would probably generate a greater stability of this disorder with the consequent identification of high-risk populations for developing dementia, and earlier possibility to onset of drug treatment. Large longitudinal studies are needed to document the evolution of these individuals ${ }^{2}$.

\section{Conclusions}

A total of 35 studies were selected in this systematic review. The prevalence of MCI ranged from $0.5 \%$ to $41.8 \%$, with the overall pooled prevalence of $17.3 \%$. This heterogeneity in the results may be a result of differences in the characteristics of the studies, such as sample age, schooling, diagnostic criteria used, and operationalization of these criteria. MCI diagnostic criteria need to be better standardized, allowing the comparison among the studies.

\section{Acknowledgements}

Ana Julia L. Bomfim is a recipient of a scholarship from the Coordination of Improvement of Higher Level Personnel/Capes, Brazil (Financial code 001).

\section{Disclosure}

The authors declare no conflict of interest.

\section{References}

1. Knopman DS, Petersen RC. Mild cognitive impairment and mild dementia: a clinical perspective. Mayo Clin Proc. 2014;89(10):1452-9.

2. Petersen RC. Mild cognitive impairment as a diagnostic entity. J Intern Med. 2004;256(3):183-94.

3. Petersen RC, Roberts RO, Knopman DS, Boeve BF, Geda YE, Ivnik $\mathrm{R}$, et al. Mild cognitive impairment: ten years later. Arch Neurol. 2009;66(12):1447-55.

4. Dawe B, Procter A, Philpot M. Concepts of mild memory impairment in the elderly and their relationship to dementia: a review.Int J Geriatr Psychiatry. 1992;7(7):473-9.

5. Luck T, Luppa M, Briel S, Riedel-Heller SG. Incidence of mild cognitive impairment: a systematic review. Dement Geriatr Cogn Disord. 2010;29(2):164-75.

6. Petersen RC, Doody R, Kurz A, Mohs RC, Morris JC, Rabins PV, et al. Current Concepts in Mild Cognitive Impairment. Arch Neurol. 2001;58(12):1985-92.

7. Ritchie K, Artero S, Touchon J. Classification criteria for mild cognitive impairment: a population-based validation study. Neurology. 2001;56(1):37-42.

8. 8.Hoy D, Brooks P, Woolf A, Blyth F, March L, Bain C, et al. Assessing risk of bias in prevalence studies: modification of an existing tool and evidence of interrater agreement. J Clin Epidemiol. 2012;65(9):934-9.

9. Gao S, Unverzagt FW, Hall KS, Lane KA, Murrell JR, Hake AM, et al.Mild Cognitive Impairment, Incidence, Progression, and Reversion: Findings from a Community-based Cohort of Elderly African Americans. Am J Geriatr Psychiat. 2014;22(7): 670-81.

10. Jager CA, Budge MM. Stability and predictability of the classification of mild cognitive impairment as assessed by episodic memory test performance over time. Neurocase. 2005;11(1):72-9.

11. Low LF, Brodaty H, Edwards R, Kochan N, Draper B, Trollor J, et al. The prevalence of "cognitive impairment no dementia" in communitydwelling elderly: a pilot study. Aust N Z J Psychiatry. 2004;38(9):725-31.

12. Olazarán J, Valentí M, Frades B, Zea-Sevilla MA, Ávila-Villanueva M, Fernández-Blázquez MÁ, et al. The Vallecas Project: A cohort to identify early markers and mechanisms of Alzheimer's disease. Front Aging Neurosci. 2015;7(181):1-10.
13. Trittschuh EH, Crane PK, Larson EB, Cholerton B, McCormick WC McCurry SM, et al. Effects of varying diagnostic criteria on prevalence of mild cognitive impairment in a community based sample. J Alzheimers Dis. 2011;25(1):163-73.

14. Jungwirth S, Weissgram S, Zehetmayer S, Tragl KH, Fischer P. VITA: Subtypes of mild cognitive impairment in a community-based cohort at the age of 75 years. Int J Geriatr Psych. 2005;20(5):452-8.

15. Artero S, Ancelin ML, Portet F, Dupuy A, Berr C, Dartigues JF, et al. Risk profiles for mild cognitive impairment and progression to dementia are gender specific. J Neurol Neurosurg Psychiatry. 2008;79(9):979-84.

16. Jia J, Zhou A, Wei C, Jia X, Wang F, Li F, et al. The prevalence of mild cognitive impairment and its etiological subtypes in elderly Chinese. Alzheimers Dement. 2013;10(4):439-47.

17. Afgin AE, Massarwa M, Schechtman E, Israeli-Korn SD, Strugatsky R, Abuful A, et al. High prevalence of mild cognitive impairment and $\mathrm{Al}-$ zheimer's disease in Arabic villages in northern Israel: impact of gender and education. J Alzheimers Dis. 2012;29(2):431-9.

18. Ding D, Zhao Q, Guo Q, Meng H, Wang B, Luo J, et al. Prevalence of mild cognitive impairment in an urban community in China: a crosssectional analysis of the Shanghai Aging Study. Alzheimers Dement. 2015;11(3):300-9.

19. Lee KS, Cheong HK, Oh BH, Na DL, Hong CH. Working criteria of mild cognitive impairment in community: findings from Gwangju Dementia and Mild Cognitive Impairment Study (GDEMCIS). Asia-Pacific Psychiatry. 2009;1(1):15-22.

20. Ogunniyi A, Adebiyi AO, Adediran AB, Olakehinde OO, Siwoku AA Prevalence estimates of major neurocognitive disorders in a rural Nigerian Community. Brain Behav. 2016;6(7): e00481.

21. Petersen RC, Roberts RO, Knopman DS, Geda YE, Cha RH, Pankratz VS, et al. Prevalence of mild cognitive impairment is higher in men The Mayo Clinic Study of Aging. Neurology. 2010;75(10):889-97.

22. Pilleron S, Jésus P, Desport JC, Mbelesso P, Ndamba-Bandzouzi B, Clément JP, et al. Association between mild cognitive impairment and dementia and undernutrition among elderly people in Central Africa: some results from the EPIDEMCA (Epidemiology of Dementia in Central Africa) programme. Br J Nutr. 2015;114(2):306-15.

23. Richard E, Reitz C, Honig LH, Schupf N, Tang MX, Manly JJ, et al. Late-life depression, mild cognitive impairment, and dementia. JAMA Neurol. 2013;70(3):374-82.

24. Tiwari SC, Srivastava G, Tripathi RK, Pandey NM, Agarwal GG, Pandey S, et al. Prevalence of psychiatric morbidity amongst the community dwelling rural older adults in northern India. Indian J Med Res. 2013;138(4):504-14.

25. Vanoh D, Shahar S, Din NC, Omar A, Vyrn CA, Razali R, et al. Predictors of poor cognitive status among older Malaysian adults: baseline findings from the LRGS TUA cohort study. Aging Clin Exp Res. 2017; 29(2):173-82.

26. Yu J, Lam CLM, Lee TMC. Perceived loneliness among older adults with mild cognitive impairment. Int Psychogeriatr. 2016;28(10):1681-5.

27. Choi SJ, Jung SS, You YS, Shin BS, Kim JE, Yoon SW, et al. Prevalence of Alzheimer's dementia and its risk factors in community-dwelling elderly Koreans. Psychiatry Investig. 2008;5(2):78-85.

28. Juarez-Cedillo T, Sanchez-Arenas R, Sanchez-Garcia S, Garcia-Penaa C, Hsiung GYR, Sepehry AA, et al. Prevalence of mild cognitive impairment and its subtypes in the Mexican population. Dement Geriatr Cogn Disord. 2012;34(5-6):271-81.

29. Katz MJ, Lipton RB, Hall CB, Zimmerman ME, Sanders AE, Verghese J, et al. Age-specific and sex-specific prevalence and incidence of mild cognitive impairment, dementia, and Alzheimer dementia in blacks and whites: a report from the Einstein Aging Study. Alzheimer Dis Assoc Disord. 2012;26(4):335-43.

30. Khedr E, Fawi G, Abbas MA, Mohammed TA, El-Fetoh NA, Al Attar G, et al. Prevalence of mild cognitive impairment and dementia among the elderly population of Qena Governorate, Upper Egypt: a communitybased study. J Alzheimers Dis. 2015;45(1):117-26.

31. Kumar R, Dear KBG, Christensen H, Ilschner S, Jorm AF, Meslin C, et al. Prevalence of mild cognitive impairment in 60 to 64 -year-old Community-dwelling individuals: The Personality and Total Health through Life 60+ Study. Dement Geriatr Cogn Disord. 2005;19(2-3):67-74.

32. Lee SB, Kim KW, Youn JC, Park JH, Lee JJ, Kim MH, et al. Prevalence of mild cognitive impairment and its subtypes are influenced by the 
application of diagnostic criteria: results from the Korean Longitudinal Study on Health and Aging (KLoSHA). Dement Geriatr Cogn Disord. 2009;28(1):23-9.

33. Lee LK, Shahar S, Chin AV, MohdYusoff NA, Rajab N, Aziz SA. Prevalence of gender disparities and predictors affecting the occurrence of mild cognitive impairment (MCI). Arch Gerontol Geriatr. 2012;54(1):185-91.

34. Louis ED, Schupf N, Manly J, Marder K, Tang MX, Mayeux R. Association between mild parkinsonian signs and mild cognitive impairment in a community. Neurology. 2005;64(7):1157-61.

35. Ma F, Wu T, Zhao J, Ji L, Song A, Zhang M, et al. Prevalence of mild cognitive impairment and its subtypes among Chinese older adults: role of vascular risk factors.Dement Geriatr Cogn Disord. 2016;41(5-6):261-72.

36. Purser JL, Fillenbaum GG, Pieper CF, Wallace RB. Mild cognitive impairment and 10-year trajectories of disability in the Iowa Established Populations for Epidemiologic Studies of the Elderly cohort. J Am Geriatr Soc. 2005;53(11):1966-72.

37. Shimada H, Makizako H, Doi T, Yoshida D, Tsutsumimoto K, Anan Y, et al. Combined prevalence of frailty and mild cognitive impairment in a population of elderly Japanese people. J Am Med Dir Assoc. 2013;14(7):518-24.

38. Wang Y, Song M, Yu L, Wang L, An C, Xun S, et al. Mild cognitive impairment: vascular risk factors in community elderly in four cities of Hebei Province, China. PLoS One. 2015;10(5):e0124566.

39. César KG, Brucki SMD, Takada LT, Nascimento LFC, Gomes CMS, Almeida MCS, et al. Prevalence of cognitive impairment without dementia and dementia in Tremembé, Brazil. Alzheimer Dis Assoc Disorde. 2016;30(3):264-71.

40. Hilal S, Ikram MK, Saini M, Tan CS, Catindig JA, Dong YH, et al. Prevalence of cognitive impairment in Chinese: epidemiology of dementia in Singapore study. J Neurol Neurosurg Psychiatry. 2013;84(6):686-92.

41. Hughes TF, Chang CCH, Bilt JV, Snitz BE, Ganguli M. Mild cognitive deficits and everyday functioning among older adults in the community: The Monongahela-Youghiogheny healthy aging team study. Am J Geriatr Psychiatry. 2012;20(10):836-44.
42. Paddick S, Kisoli A, Samuel M, Higginson J, Gray WK, Dotchin CL, et al. Mild Cognitive Impairment in Rural Tanzania: Prevalence, Profile, and Outcomes at 4-Year Follow-up. Am J Geriatr Psychiatry. 2015;23(9):950-9.

43. Saxton J, Snitz BE, Lopez OL, Ives DG, Dunn LO, Fitzpatrick A, et al. Functional and cognitive criteria produce different rates of mild cognitive impairment and conversion to dementia. J Neurol Neurosurg Psychiatry. 2009;80(7):737-43.

44. Trittschuh EH, Crane PK, Larson EB, Cholerton B, McCormick WC, McCurry SM, et al. Effects of varying diagnostic criteria on prevalence of mild cognitive impairment in a community based sample. J Alzheimers Dis. 2011;25(1):163-73.

45. Luck T, Riedel-Heller SG, Kaduszkiewicz H, Bickel H, Jessen F, Pentzek $\mathrm{M}$, et al. Mild cognitive impairment in general practice: age-specific prevalence and correlate results from the German study on ageing, cognition and dementia in primary care patients (AgeCoDe).Dement Geriatr Cogn Disord. 2007;24(4):307-16.

46. Ivnik R, Smith G, Lucas J, Petersen RC, Boeve BF, Kokmen E, et al. Testing normal older people three or four times at 1- to 2-year intervals: defining normal variance. Neuropsychology. 1999;13:121-7.

47. Bickel H, Mösch E, Seigerschmidt E, Siemen H, Förstl H. Prevalence and persistence of mild cognitive impairment among elderly patients in general hospitals. Dement Geriatr Cogn Disord. 2006;21(4):242-50.

48. Albert MS, DeKosky ST, Dickson D, Dubois B, Feldman HH, Fox NC, et al. The diagnosis of mild cognitive impairment due to Alzheimer's disease: Recommendations from the National Institute on Aging-Alzheimer's Association workgroups on diagnostic guidelines for Alzheimer's disease. Alzheimers Dement. 2011;7(3):270-9.

49. Jak AJ, Bondi MW, Delano-Wood L, Wierenga C, Corey-Bloom J, Salmon DP, et al. Quantification of five neuropsychological approaches to defining mild cognitive impairment. Am J Geriatr Psychiatry. 2009;17(5):368-75. 